\title{
Interaction famille $\times$ site dans un test tristationnel de descendances de Douglas (Pseudotsuga menziesii (Mirb.) Franco)
}

\author{
C.-E. DUREL et B. ROMAN-AMAT \\ INRA, Station d'Amélioration des Arbres forestiers \\ Ardon. F 45160 Olivet
}

\begin{abstract}
Résumé
Cet article analyse l'interaction génotype $\times$ environnement dans un groupe de 63 descendances maternelles issues de provenances françaises de Douglas. Sur trois sites expérimentaux très contrastés, six caractères différents de hauteur, phénologie et forme sont étudiés. Après analyse de variance, les écovalences de WRicke (1962) et leurs inverses, appelées interactivités. sont calculées pour les sites et les familles sur les quatre caractères présentant une forte interaction. Une étude de ces interactivités est réalisée : distribution, corrélations entre elles, corrélations avec les performances familiales. L'évolution du $F$ d'interaction pour la hauteur totale est observée au cours de l'élimination des familles par ordre d'interactivités décroissantes. Pour ce caractère, un tiers des familles sont responsables des trois quarts de l'interactivité globale. Un groupe de familles stables dans l'espace est ainsi identifié. II apparaît que la moyenne et les variances de ce caractère restent stables lorsque le nombre de familles diminue. Une démarche similaire est tentée avec une interactivité synthétique multicaractères. L'utilité des interactivités pour l'améliorateur est enfin brièvement discutée.
\end{abstract}

Mots clés: Interaction génotype-environnement, descendance maternelle, croissance hauteur, débourrement végétatif, forme du fût, angle des branches, Pseudotsuga menziesii.

\section{Introduction}

La phase d'exploration de la variabilité entre provenances touchant à sa fin, le programme INRA d'amélioration du Douglas accorde actuellement une place croissante à la sélection familiale et individuelle. Or, de manière générale, plus la base génétique du matériel étudié est étroite, plus les risques de manifestation d'une interaction entre les génotypes et les milieux sont élevés.

De nombreuses méthodes d'analyse statistique de l'interaction "génotype $\times$ environnement " ont été proposées dans la littérature. Quelques auteurs, dont notamment Freeman (1973) puis Denis \& Vincourt (1982), en ont réalisé des synthèses bibliographiques. En amélioration des arbres forestiers, le nombre de sites expérimentaux est en général réduit pour des raisons matérielles (disponibilités en nombre de plants, en surfaces, coûts). En conséquence, les méthodes utilisant les régressions des performances génotypiques sur les moyennes de sites, très abondamment analysées dans la 
littérature, sont rarement adaptées, comme l'indique SKRфPPA (1984). En revanche, l'analyse de variance complétée par le calcul dés écovalences de WRicke (1962) est toujours utilisable. Un exemple de mise en application de cette technique est présenté dans cet article. Deux objectifs sont poursuivis :

- mettre en évidence la présence éventuelle: de l'interaction sur différents caractères importants pour l'améliorateur, et l'analyser ;

- rechercher une méthode pratique permettant de constituer un groupe de génotypes stables dans l'espace pour un ou plusieurs caractères.

L'accent est davantage placé sur les méthodes d'analyse que sur les résultats expérimentaux. En outre, l'approche est volontairement plus statistique que génétique.

\section{Matériels et méthodes}

\subsection{Matériel végétal}

L'expérience analysée est un test multistationnel de provenances françaises de Douglas représentées chacune par quelques descendances maternelles récoltées en forêt. Le niveau provenance est volontairement ignoré. Le matériel végétal étudié est décrit dans le tableau 1.

TABLEAU 1

Provenances et familles expérimentées.

Provenances and families in test.

\begin{tabular}{|c|c|c|}
\hline Provenances & Département & $\begin{array}{c}\text { Nombre } \\
\text { de Familles }\end{array}$ \\
\hline En Argaud. & Saône-et-Loire & 5 \\
\hline Bois Chicard & " & 2 \\
\hline Bois des Cusses & $"$ & 3 \\
\hline Bois du Moulin . & $"$ & 2 \\
\hline Bois rond $\ldots$. & $"$ & 2 \\
\hline Bruyères de Pormmey . . . . . . . & $"$ & 4 \\
\hline Au Charnay ........... & $"$ & 1 \\
\hline Chassagne $\ldots \ldots \ldots \ldots$ & Rhône & 4 \\
\hline Chevenatte $\ldots \ldots \ldots \ldots$ & $"$ & 5 \\
\hline Les Communes $\ldots \ldots \ldots \ldots$ & Sitône-et-Loire & 4 \\
\hline Etang Bonnin $\ldots \ldots \ldots \ldots$ & " & 2 \\
\hline La Garenne $\ldots \ldots \ldots \ldots$ & $"$ & 2 \\
\hline Les Genievriers $\ldots \ldots \ldots$ & $"$ & 3 \\
\hline La Gravière $\ldots \ldots \ldots \ldots$ & $"$ & 2 \\
\hline Marcon I ........... & $"$ & 1 \\
\hline$\ldots \ldots \ldots \ldots$ & Héraul: & 2 \\
\hline$\ldots \ldots \ldots \ldots$ & Rhône & 2 \\
\hline$\ldots \ldots \ldots$ & Loire & 2 \\
\hline$\ldots \ldots \ldots \ldots$ & Rhônc & 6 \\
\hline Aux Pralles. & Sâone-et-Loire & 1 \\
\hline$\ldots \ldots \ldots \ldots$ & $"$ & 2 \\
\hline Les Serrets $\ldots \ldots \ldots \ldots \ldots$ & Rhône & 2 \\
\hline Villars I . . . . . . . . & $"$ & 2 \\
\hline Villars II $\ldots \ldots \ldots \ldots$ & $"$ & 1 \\
\hline
\end{tabular}




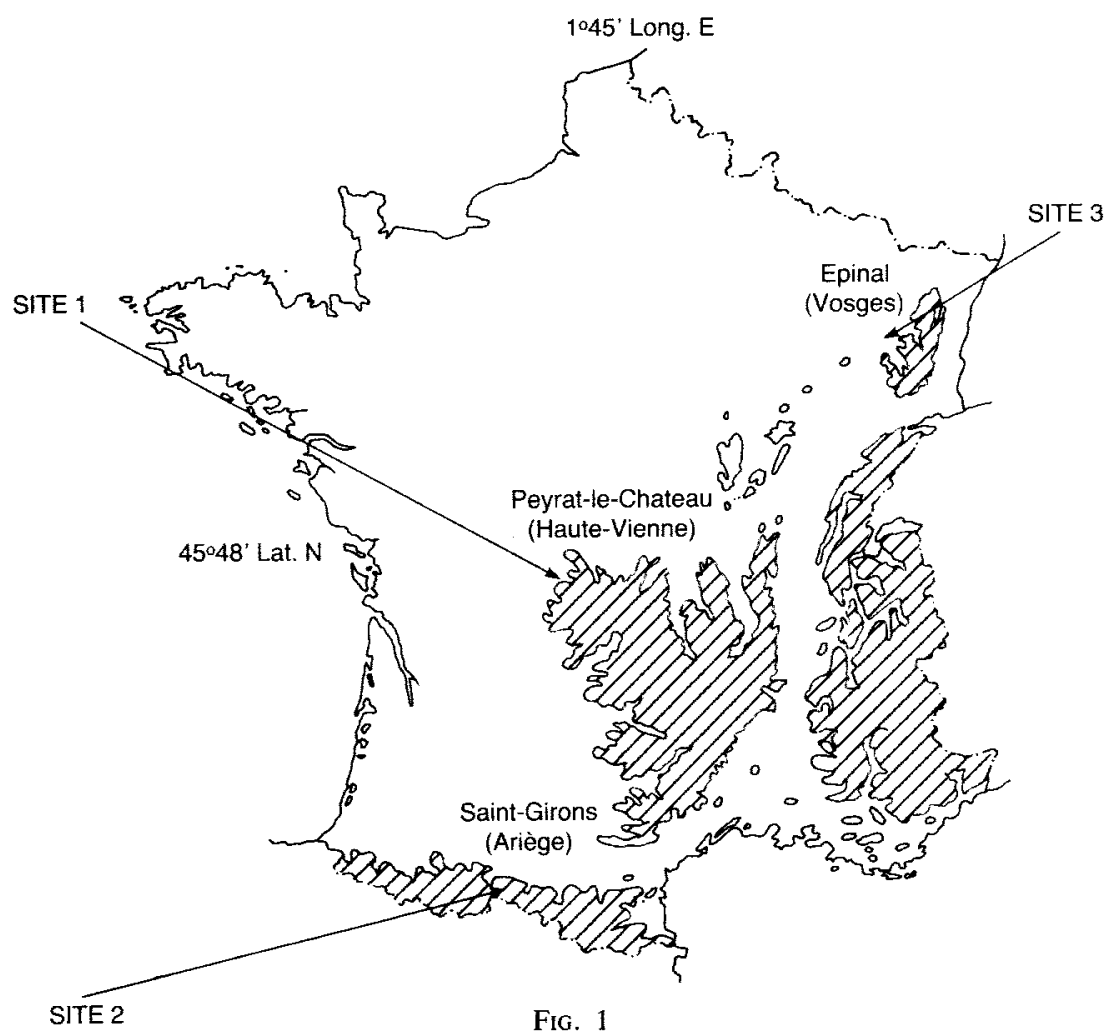

Localisation et caractéristiques des dispositifs expérimentaux.

Location and features of field trials.

\begin{tabular}{|c|c|c|c|}
\hline & $\begin{array}{c}\text { Site } 1 \\
\text { Peyrat-le-Château }\end{array}$ & $\begin{array}{c}\text { Site } 2 \\
\text { Saint-Girons }\end{array}$ & $\begin{array}{l}\text { Site } 3 \\
\text { Epinal }\end{array}$ \\
\hline - Altitude $\ldots \ldots \ldots \ldots \ldots$ & $450 \mathrm{~m}$ & $900 \mathrm{~m}$ & $400 \mathrm{~m}$ \\
\hline $\begin{array}{l}\text { - Pluviométrie annuelle } \ldots . . . \\
\text { Annual rainfall }\end{array}$ & $1130 \mathrm{~mm}$ & $1800 \mathrm{~mm}$ & $1000 \mathrm{~mm}$ \\
\hline $\begin{array}{c}\text { - Type de sol } \ldots \ldots \ldots \ldots \ldots \\
\text { Soil type }\end{array}$ & $\begin{array}{c}\text { brun humifère } \\
\text { sur arène granitique }\end{array}$ & $\begin{array}{l}\text { brun peu épais } \\
\text { sur schiste }\end{array}$ & $\begin{array}{l}\text { brun lessivé } \\
\text { sur gravier et sable }\end{array}$ \\
\hline $\begin{array}{l}\text { - Espacement de plantation } . . . \\
\text { Tree spacing }\end{array}$ & $2 \mathrm{~m} \times 1,5 \mathrm{~m}$ & $2 \mathrm{~m} \times 2 \mathrm{~m}$ & $2,5 \mathrm{~m} \times 2,5 \mathrm{~m}$ \\
\hline $\begin{array}{l}\text { - Dispositif } \\
\text { Lay-out }\end{array}$ & & & \\
\hline $\begin{array}{l}\text { - Nombre de familles } \\
\text { Number of families }\end{array}=\mathrm{t} \ldots$. & $t=64$ & $\mathrm{t}=125$ & $t=125$ \\
\hline $\begin{array}{l}\text { Nombre de blocs } \\
\text { Number of blocks }=\mathrm{b} \ldots \ldots\end{array}$ & $b=48$ & $\mathrm{~b}=75$ & $\mathrm{~b}=75$ \\
\hline $\begin{array}{l}\text { - Nombre de répétitions } \\
\text { Number of replicates }\end{array}=\mathrm{r} \ldots$ & $r=3$ & $r=3$ & $r=3$ \\
\hline $\begin{array}{l}\text { Nombre de plants } \\
\text { par parcelle unitaire } \\
\text { Number of trees per plot }=\text { p.u. }\end{array}$ & p.u. $=16$ & p.u. $=16$ & p.u. $=16$ \\
\hline
\end{tabular}




\subsection{Dispositifs expérimentaux}

Les trois dispositifs expérimentaux analysés ont été installés au printemps 1978 à l'aide de plants de 3 ans. Leurs caractéristiques principales sont indiquées sur la figure 1. Ces trois plantations sont très éloignées les unes des autres des points de vue géographique et écologique, mais chacune d'elles correspond à un site normal ou potentiel d'introduction du Douglas.

\subsection{Caractères étudiés}

Six caractères considérés habituellement comme importants pour le Douglas ont été enregistrés sur tous les individus :

a) la hauteur totale $(\mathrm{cm})$ à la fín de la saison 1984 , soit 7 ans après plantation ou 10 ans après la graine ;

b) le débourrement végétatif, en nombre de jours depuis une origine arbitraire, enregistré en 1981 à Peyrat-le-Château et Epinal, et en 1984 à Saint-Girons. Pour ce caractère, le facteur "site " englobe donc les effets climatiques des printemps 1981 ou 1984 selon les cas. Cependant, il semble possible d'accepter l'hypothèse de la stabilité du classement des familles pour le débourrement végétatif dans un site donné quelle que soit l'année ; 2a) ;

c) la flexuosité du fût, notée fin 1984 sur larbre entier par une note de 1 à 5 (fig.

d) l'angle d'insertion des branches, évalué fin 1984 sur l'arbre entier par une note de 0 à 4 (fig. 2b) ;
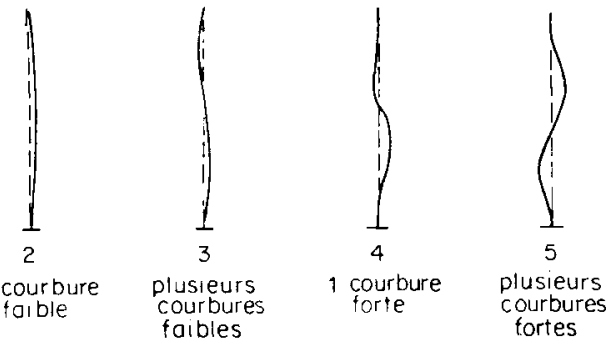

2a: Stem sinuosity (on the whole tree)

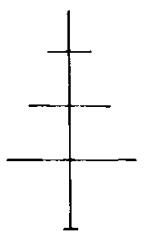

0

horizontal

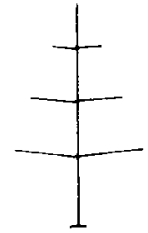

sub-horizontal intermediare

2

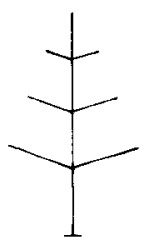

2b: angle d'insertion des branches

2b: branche angle

Fig. 2
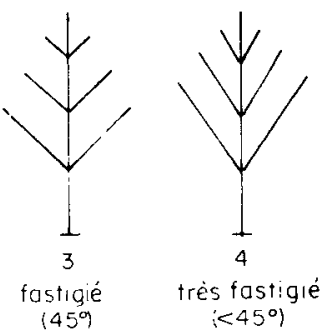

Echelles de cotation employées pour les caractères de forme du fût (2a) et des branches (2b). Scales used for form traits of stem (2x) and branches (2b). 
e) la fourchaison, appréciée fin 1984 par une note inférieure ou égale à 200 , fonction de l'abondance de fourches $\left({ }^{*}\right)$ et des ramicornes $\left({ }^{*}\right)$;

f) la présence ou l'absence de pousse d'août sur la pousse principale de 1984, notée 1 ou 0 respectivement.

\subsection{Méthodes d'analyse statistique}

Modèles d'analyse de variance :

Une analyse préalable a été effectuée indépendamment pour chaque site, selon le modèle suivant :

$$
\mathrm{Xijk}=\mu+\mathrm{fi}+\mathrm{bj}+\varepsilon \mathrm{ijk}
$$

$\mathrm{Xijk}=$ valeur du caractère $\mathrm{X}$ de l'individu $\mathrm{k}$ appartenant à la famille $\mathrm{i}$ présente dans le bloc $\mathrm{j}$;

$\mu \quad=$ moyenne générale ;

fi $=$ effet moyen de la famille $\mathbf{i}$;

bj $\quad=$ effet moyen du bloc $\mathrm{j}$;

$\varepsilon i j k=$ erreur résiduelle aléatoire.

Les valeurs de départ (Xijk) ont ensuite été ajustées aux blocs pour chaque site, d'où le modèle :

$$
X^{\prime} i k=\mu+f i+\varepsilon i k
$$

$\mathrm{X}$ 'ik = valeur du caractère $\mathrm{X}$ de l'individu $\mathrm{k}$ appartenant à la famille $\mathrm{i}$ ajustée à l'effet bloc;

cik = erreur résiduelle aléatoire.

Pour les analyses de variance multisites (respectivement 2 et 3 sites), le modèle suivant a été appliqué :

$$
\mathrm{X}^{\prime} \mathrm{ilk}=\mathrm{M}+\mathrm{Fi}+\mathrm{Sl}+(\mathbf{F} \times \mathrm{S}) \mathrm{il}+\text { eilk }
$$

X'ilk = valeur (ajustée au bloc) du caractère $\mathrm{X}$ de l'individu $\mathrm{k}$ appartenant à la famille $\mathrm{i}$ présente dans le site 1 ;

$\mathrm{M} \quad=$ moyenne générale,

$\mathrm{Fi} \quad=$ effet moyen de la famille $\mathrm{i}$,

SI = effet moyen du site $\mathbf{I}$,

$(F \times S) i l=$ effet moyen d'interaction entre famille $\mathrm{i}$ et site 1

zilk = erreur résiduelle aléatoire.

Le facteur «famille» a toujours été considéré comme aléatoire, les facteurs "bloc" et " site" comme fixes.

\subsection{Calcul d'interactivités}

Après les analyses de variance multisites, il est possible de mesurer la participation de chaque niveau de chaque facteur à la valeur estimée du carré moyen d'interaction.

(*) Définies comme des branches à angle d'insertion inféricur à $30^{\circ}$ dont le diamètre est respectivement voisin ou neltement inférieur à celui de la tige principale. On affecte la note 200 à un arbre sans défaut, et on retire 5 points par ramicorne et 10 points par fourche. 
en calculant des paramètres d'interactivité. Une famille ou un site dont l'interactivité, définie comme l'inverse de l'écovalence de WRICKE (1962), est forte (respectivement faible) a un poids important (respectivement faible) dans la valeur totale du carré moyen d'interaction.

Pour la famille i, la valeur de l'interactivité est la suivante :

$\mathrm{Ii}=\frac{1}{\text { ni... }} \times \sum_{\mathrm{I}=1}^{\mathrm{s}}$ nil. $(\widehat{\mathrm{F} \times \mathrm{S}})^{2}$ il

Ii $=$ interactivité brute de la famille $\mathbf{i}$;

$(\widehat{F \times S})$ il = valeur estimée de l'effet d'interaction entre la famille $i$ et le site 1 ;

nil. $\quad=$ nombre d'individus de la famille $\mathrm{i}$ présents dans le site 1 ;

ni.. $\quad=$ nombre total d'individus de la famille $\mathrm{i}$ (sur l'ensemble des sites) ;

$\mathrm{s}=$ nombre total de sites.

La somme des interactivités brutes étant arbitrairement fixée égale à 100 , il est possible de calculer pour chaque famille une interactivité relative d'après la formule :

$$
\mathrm{IRi}=\frac{100 \times \mathrm{Ii}}{\mathrm{u} \stackrel{\mathrm{f}}{=} \mathrm{Iu}}
$$

$\mathrm{IR} \mathrm{i}=$ interactivité relative la famille $\mathrm{i}$;

$\mathrm{f}=$ nombre total de familles.

Les interactivités relatives présentent l'avantage d'être très facilement comparables entre elles.

Le même type de calcul a été appliqué pour les interactivités des sites.

\subsection{Interactivités synthétiques}

Pour chaque famille, une interactivité synthétique a été calculée pour représenter son instabilité pour 2 caractères simultanément, selon la formule suivante :

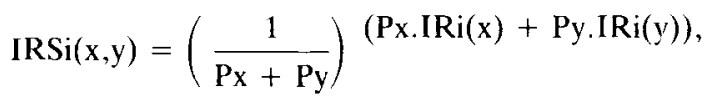

$\operatorname{IRSi}(x, y)=$ interactivité relative synthétique de la famille i pour les 2 caractères $\mathrm{x}$ et $y$,

$\operatorname{IRi}(\mathrm{x})=$ interactivité relative de la famille $\mathrm{i}$ pour le caractère $\mathrm{x}$,

Px $=$ coefficient de pondération choisi pour le caractère $\mathrm{x}$.

Cette interactivité synthétique est une simple moyenne pondérée d'interactivités monocaractères. Elle n'est utilisable en pratique que lorsque ces dernières sont indépendantes les unes des autres. Elle est généralisable au cas de plus de deux caractères.

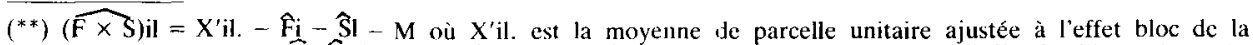
famille $i$ dans le site 1 ct $\widehat{F}$, Ŝl les estimations aux moindre's carrés respectivement de l'effet famille et de l'cffet site, telles que $\sum_{i}$ ni... $\hat{F i}=\sum_{i}$ n.I.SI $=0$. 
Il a été décidé d'attribuer à chaque coefficient de pondération la valeur du test $\mathrm{F}$ d'interaction du caractère correspondant. De la sorte, l'interactivité relative synthétique tient compte du niveau relatif d'interaction constaté sur chacun des deux caractères.

N.B. Tous les calculs ont été effectués à l'aide de la programmathèque AMANCE (BACHACOU et al., 1981).

\section{Résultats}

\subsection{Analyse site par site}

Les résultats des analyses de variance faites site par site sont présentés au tableau 2 .

- Les moyennes générales des caractères sont très différentes d'un site à l'autre (cf. en particulier la hauteur). L'existence de telles variations était prévisible d'après les conditions de milieu très contrastées des 3 stations expérimentales. Cette situation est un élément favorable pour révéler une éventuelle interaction famille $\times$ site.

- L'effet du facteur famille est variable suivant le caractère considéré. Il est très nettement significatif pour hauteur et débourrement, significatif pour flexuosité du fût et angle des branches, et moins net pour la fréquence de pousse d'août et surtout pour la fourchaison. On peut s'attendre à ce que des caractères aussi différents expriment des réactions variées vis-à-vis de l'interaction génotype $\times$ milieu.

Par ailleurs, et pour un caractère donné, la variance interfamilles peut varier fortement d'un site à l'autre. Par exemple, pour la hauteur, la valeur estimée de cette variance dans le site 3 est nettement plus grande que dans les sites 1 et 2 alors que les carrés moyens résiduels sont comparables. Dans ce cas, le site 3 est celui qui révèle le mieux les différences entre familles.

Deux types de corrélations entre les caractères soumis à un fort effet famille sont présentés dans le tableau 3:

- les corrélations purement phénotypiques, calculées sur l'ensemble des individus sans tenir compte du niveau familial. Celles de ces corrélations qui concernent la hauteur varient assez fortement d'un site à l'autre ;

- les corrélations génétiques additives estimées d'après l'analyse de covariance, et appelées «corrélations génétiques " (voir BARADAT, 1982, pour précisions). Pour les valeurs les plus significatives des coefficients de corrélation [couples (hauteur-flexuosité) et (flexuosité-angle)] leur intervalle de confiance a été calculé (FAlConer, 1974). Deux coefficients de corrélation dont les intervalles de confiance ne se recouvrent, pas peuvent être considérés comme différents. Pour les deux couples étudiés, cette méthode conduit à ne pas distinguer les valeurs des corrélations obtenues dans les sites 1 et 2 . Par contre, les valeurs du site 3 s'écartent nettement des autres.

Il est intéressant de remarquer que des liaisons génétiques entre caractères peuvent varier d'un site à l'autre. L'interaction génotype $\times$ milieu pourrait être une cause importante de ces variations. 


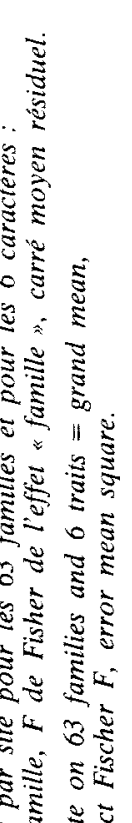

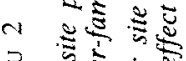

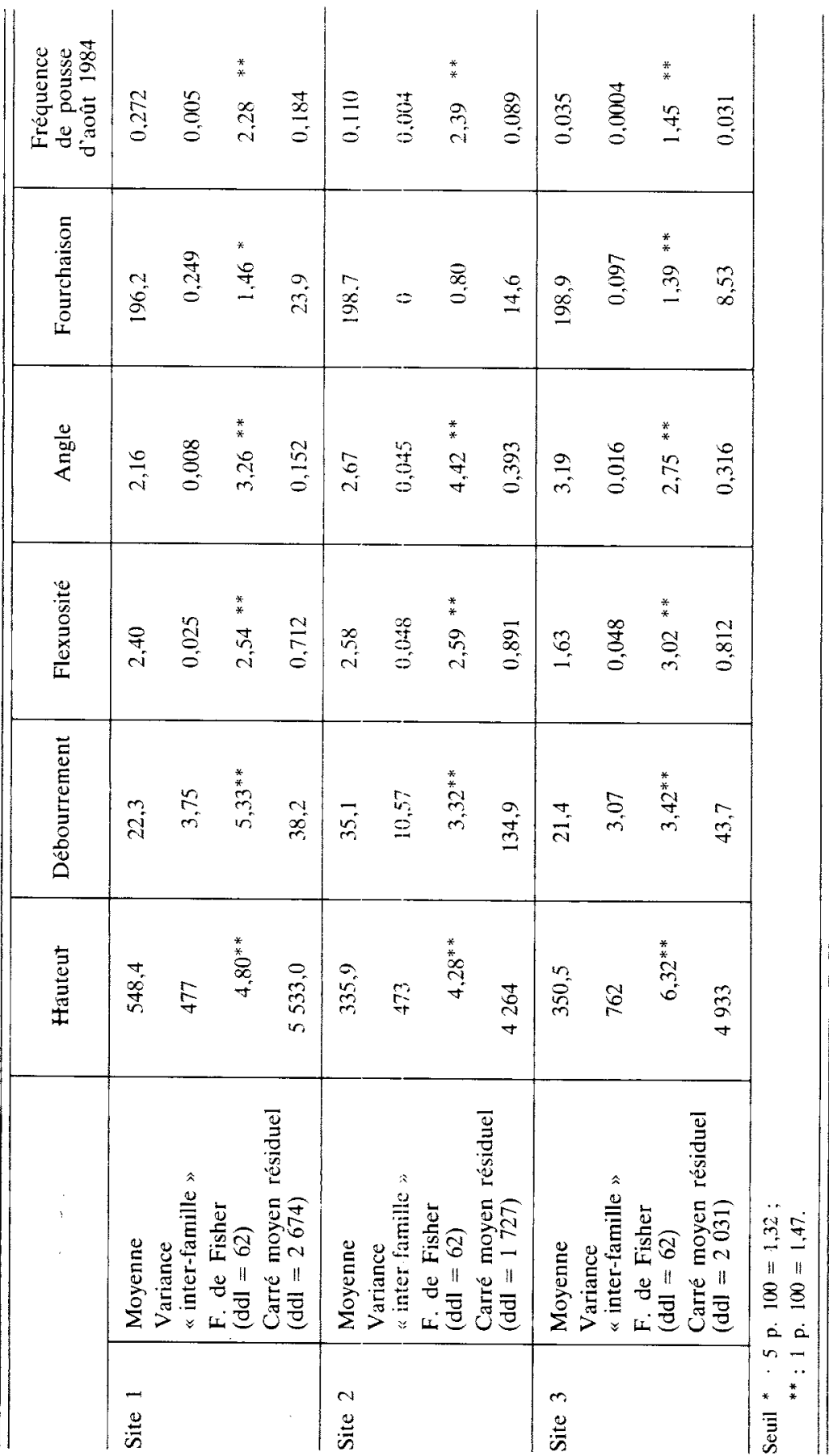




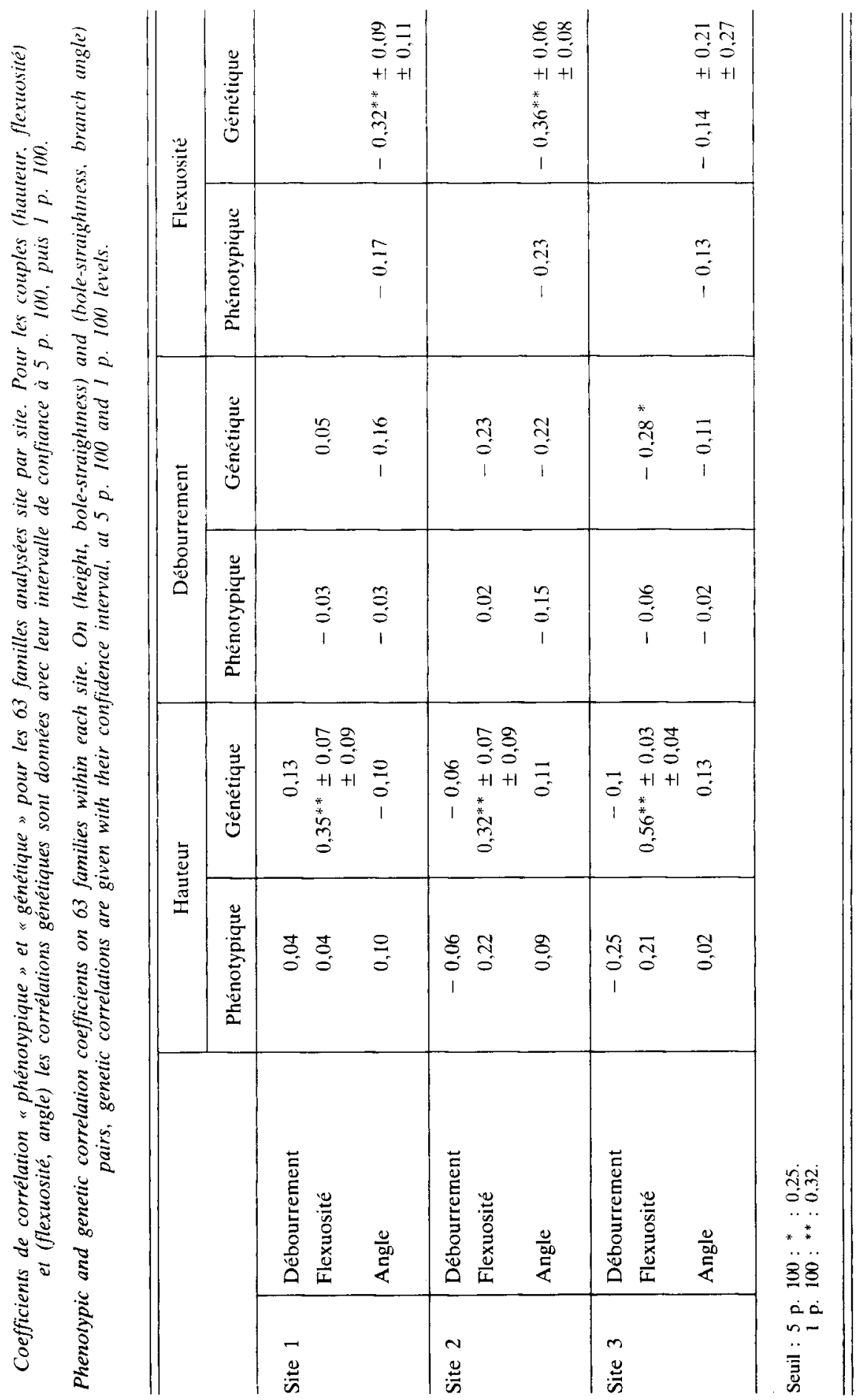




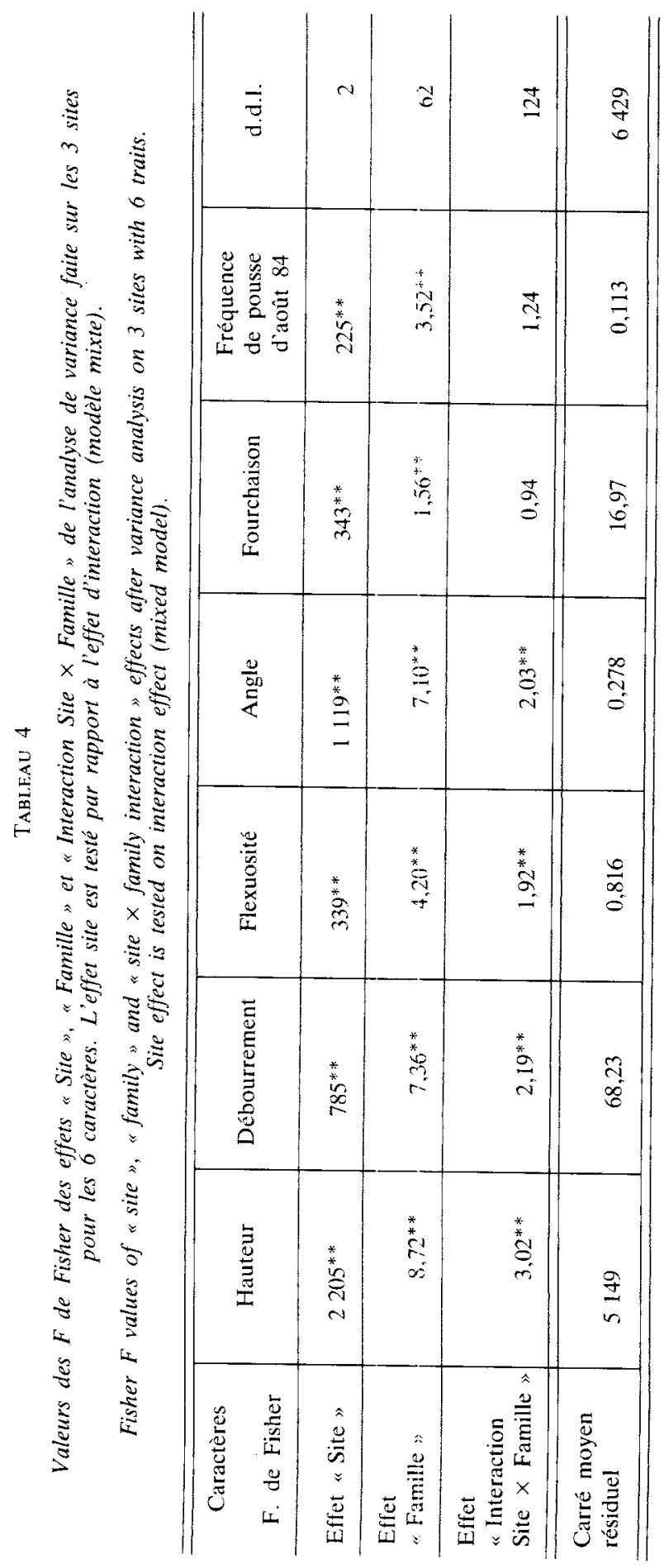




\subsection{Etude de l'interaction famille $\times$ site}

\subsection{Approche statistique}

Le modèle (3) appliqué aux 3 sites et aux 6 caractères conduit aux résultats du tableau 4:

- Bien que les valeurs du F de Fisher soient plus élevées, la hiérarchie des effets du facteur «famille» est très comparable à celle obtenue à l'intérieur des différents sites ;

- Les effets du facteur "site " sont toujours très nettement supérieurs à ceux des autres facteurs. Outre les différences écologiques entre stations expérimentales, déjà mentionnées, l'importance de ces effets est liée à la grande taille de l'échantillon et à l'utilisation de valeurs ajustées aux blocs, ce qui efface une part de la variabilité intrasite.

- Les effets d'interaction sont hautement significatifs pour les 4 premiers caractères. Dans ces cas, le carré moyen d'interaction atteint des valeurs élevées puisqu'il n'est jamais inférieur à 30 p. 100 du carré moyen «famille ». En revanche, l'effet d'interaction est non significatif pour la fourchaison et la fréquence de pousse d'août. C'est d'ailleurs sur ces deux caractères que l'effet famille se trouve être le plus faible à la fois dans chaque site et dans l'analyse multisite. Par la suite, ces deux caractères ne seront plus considérés.

\subsection{Utilisation des interactivités}

Pour les caractères présentant une interaction significative au seuil de 1 p. 100 , les interactivités relatives des sites et des familles ont été calculées.

\subsection{Interactivités des sites}

Une participation équivalente des 3 sites à l'interaction globale correspondrait à des interactivités relatives voisines de 33 p. 100 pour chacun d'eux. Cette situation ne se rencontre pour aucun des quatre caractères du tableau 5. Par exemple, pour le débourrement, le site 2 fournit à lui seul 63 p. 100 de l'interaction. En outre, un même site peut posséder une interactivité forte pour un caractère et faible pour un autre (site 3).

Quel que soit le caractère, l'interactivité relative du site 1 est toujours pratiquement la plus faible. Ce site participe donc moins à l'interaction globale que les deux autres. Cependant, lorsque le carré moyen d'interaction est très hautement significatif, le classement des familles dans un site dont l'interactivité relative n'est que de 20 p. 100 peut déjà être très différent de celui réalisé sur l'ensemble de l'expérience.

\subsection{Interactivité des familles}

En raison du nombre élevé d'interactivités calculées, seule leur distribution pour chaque caractère est présentée sur la figure 3. Si toutes les familles étaient également interactives, ces distributions seraient uniformes, chaque famille ayant une interactivité relative voisine de 1,6 p. 100 . En fait, pour chaque caractère, deux groupes de familles peuvent toujours être distingués : 


\section{TABleau 5}

Interactivités (en p. 100) des 3 sites pour les 4 caractères présentant une interaction nettement significative.

Interactivities $(p .100)$ of the 3 sites on the 4 traits on which family $\times$ site interaction is highly significant.

\begin{tabular}{c|c|c|c|c}
\hline Site & Hauteur & Débourrement & Flexuosité & Angle \\
\hline 1 & 20,5 & 19.7 & 18,2 & 22,6 \\
2 & 31,3 & 63,0 & 40,0 & 51,2 \\
3 & 48,2 & 17.3 & 41,8 & 26,2 \\
\hline Total & 100,0 & 100.0 & 100,0 & 100,0 \\
\hline
\end{tabular}
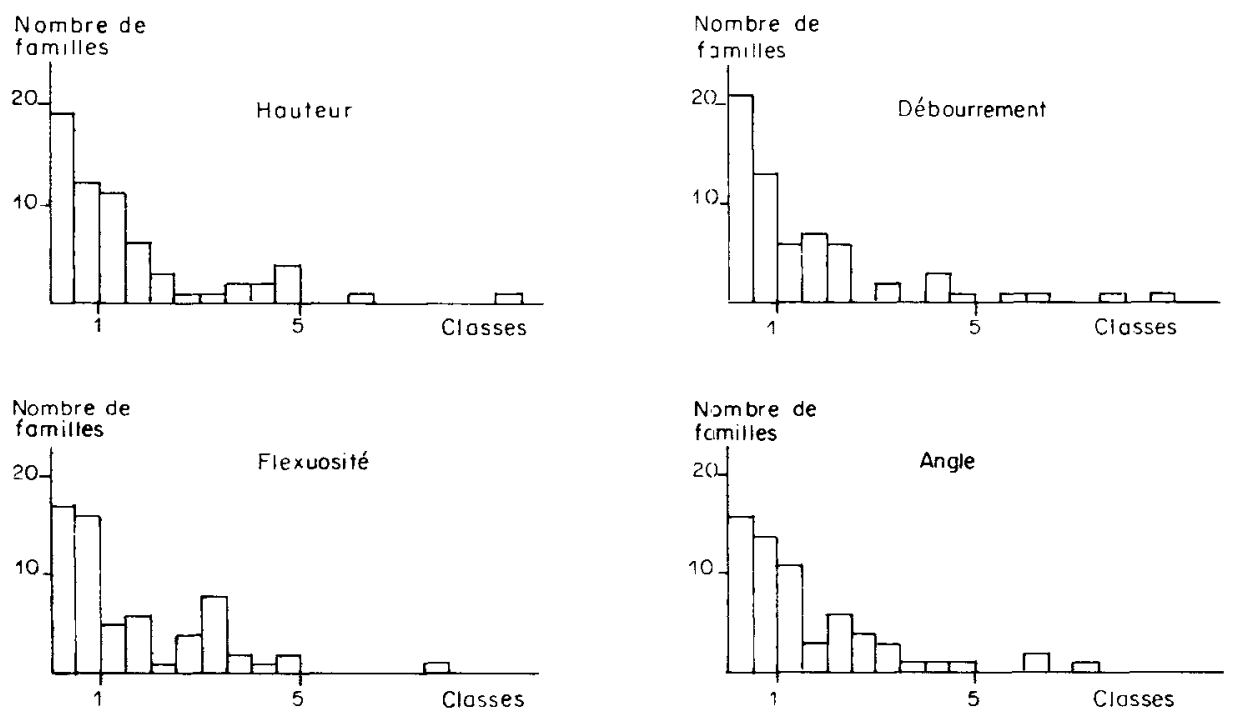

Fig. 3

Distribution de: familles

en classes d'interactivité relative de $0.5 p$. 100 d'amplitude pour 4 caractères.

Distribution of families into 0,5 p. 100 wide classes according to their relative interactivity for four traits.

- un groupe majoritaire de familles à interactivités relatives faibles à moyennes, inférieures à 2 p. 100 ;

- un groupe minoritaire de familles à interactivités relatives fortes à très fortes, supérieures à 4 p. 100 .

\section{- Corrélations entre interactivités}

Pour chaque couple de caractères, la valeur de la corrélation entre les deux groupes d'interactivités familiales est calculée (tab: 6). Aucune corrélation n'est signifi- 
cative. Les interactivités d'une même famille pour différents caractères sont donc indépendantes entre elles. Cette indépendance se constate même pour des caractères assez fortement corrélés entre eux du point de vue génétique comme hauteur et flexuosité du fût.

\section{TABleau 6}

Coefficients de corrélation entre les interactivités des familles pour les différents caractères présentant une interaction nettement significative.

Correlation coefficients between family interactivities for the 4 traits on which family $\times$ site interaction is highly significant.

\begin{tabular}{l|c|c|c}
\hline & Hauteur & Débourrement & Flexuosité \\
\hline Débourrement . . . . & 0,018 & \\
Flexuosité . . . . . . . . . . . & 0,036 & 0,017 & \\
Angle $\ldots . .0,154$ & $-0,031$ & 0,003 \\
\hline
\end{tabular}

Seuil : 5 p. $100: 0,25$

1 p. $100: 0,32$.

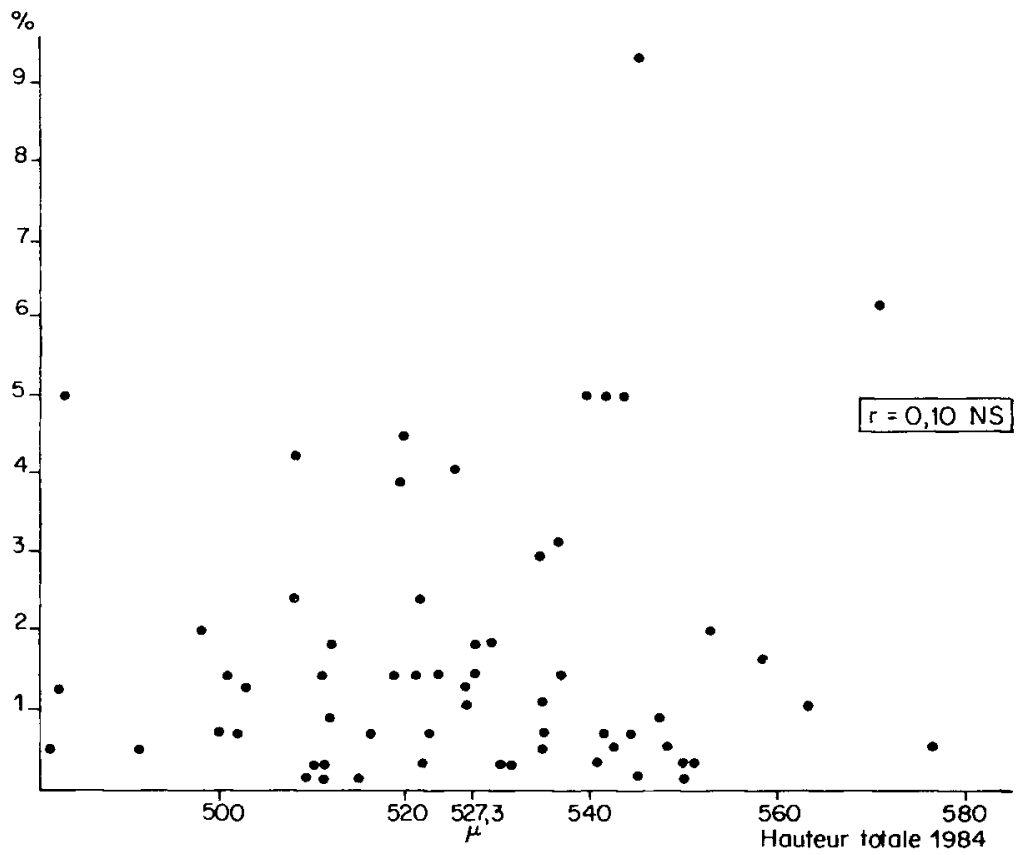

FiG. 4

Liaison entre la hauteur totale 1984 des familles en abscisses, et en ordonnées leur interactivité relative (en p. 100) pour le même caractère.

Relationship between family total height in 1984 (abscissae) and relative interactivity (p. 100, ordinates) for the same trait. 


\section{- Liaisons entre interactivités et performances des familles}

Interactivités et performances des familles peuvent être liées. L'élimination des familles à fortes interactivités risque alors d'entraîner une évolution de la moyenne générale et de la variance entre familles.

La figure 4 présente, pour la hauteur, l'interactivité relative des 63 familles en fonction de leur performance moyenne sur les 3 sites. La corrélation entre ces deux variables est non significative. Il en est de même pour les trois autres caractères. Dans ce cas particulier, l'élimination des familles les plıs interactives ne conduira donc pas à une évolution importante de la moyenne des caractères étudiés.

- Evolution du $F$ d'interaction en fonction du nombre de familles

Certaines familles contribuent plus que d'autres à l'interaction. Leur suppression permet de faire diminuer le carré moyen d'interaction et aussi la valeur correspondante du test de Fisher dans le cas où, simultanément. le carré moyen d'erreur reste stable ou augmente.

Les 63 familles ont été classées par interactivité croissante pour le caractère hauteur, ce qui a permis d'éliminer une par une les plus interactives d'entre-elles. Après chaque élimination, une nouvelle analyse de variance a été réalisée sur le groupe de familles restantes, et la nouvelle valeur du $F$ d'interaction a été relevée. Les résultats obtenus sont présentés sur la figure 5. La. valeur du F d'interaction diminue de moitié après la suppression des 14 familles les plus interactives. Elle devient non significative au seuil de 5 p. 100 lorsqu'il ne reste plus que les 42 familles les moins interactives. Les 21 familles retirées représentent à elles seules 74 p. 100 de l'interactivité globale initiale.

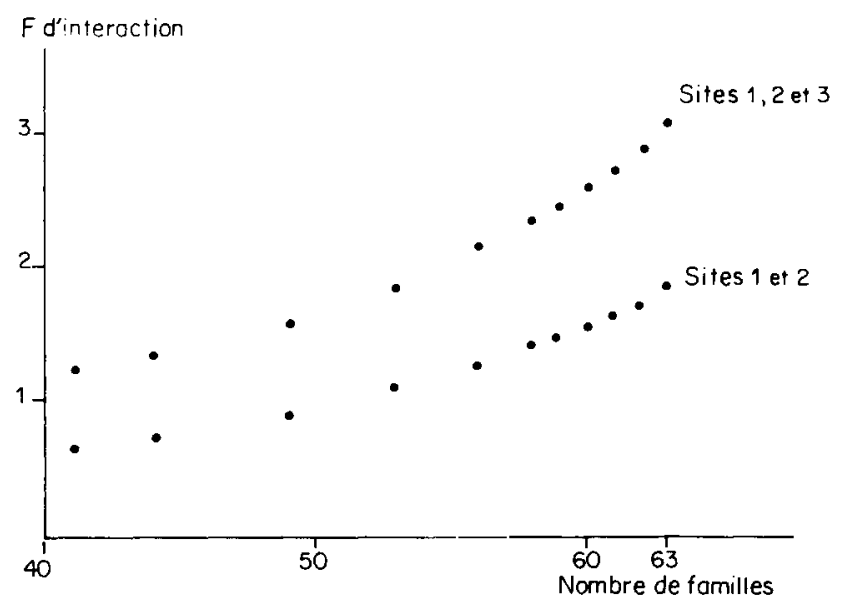

Fig. 5

Evolution du test de Fisher sur l'interaction "Famille $\times$ Site " en fonction du nombre de familles classées par interactivité décroissante (de droite à gauche) pour la hauteur 1984, dans les cas "sites 1,2 et 3 " et "sites 1 et 2 ".

N.B. : Le classement des familles n'est pas le même dans les 2 cas.

Evolution of family $\times$ sile interaction Fisher test (ordinates) in terms of the number of families (abscissae). The families are arranged in descending order of total height 1984 relative interactivity. N.B. : The ranking of families is not the same in both cases. 
Sur la figure 6, les mêmes valeurs des $\mathrm{F}$ figurent en ordonnées. En abscisses sont portées les valeurs cumulées des interactivités familiales calculées pour le groupe des 63 familles. Ces valeurs ont été obtenues en retranchant une à une à l'interactivité totale (qui vaut 100) les interactivités relatives des familles classées par valeurs décroissantes.

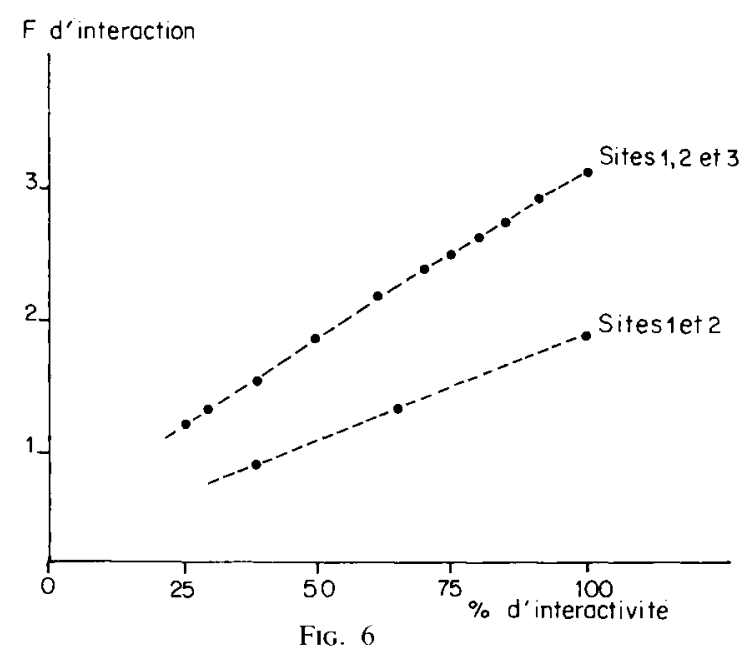

Evolution du test de Fisher sur l'interaction "Famille $\times$ Site " en fonction du pourcentage cumulé d'interactivité, dans les cas "sites 1,2 et 3 " et a sites 1 et 2 ", pour la hauteur totale 1984.

Evolution of family $\times$ site interaction Fisher test (ordinates) in terms of cumulative percentage of relaive interactivity on total height 1984 .

La liaison entre le $\mathrm{F}$ d'interaction et le pourcentage d'interactivité cumulée est presque parfaitement linéaire. Cette linéarité tient à deux causes :

- la corrélation parfaite qui existe par construction entre le pourcentage cumulé d'interactivité et la somme des carrés d'interaction ;

- la très bonne stabilité dans ce cas particulier du carré moyen résiduel quel que soit le nombre de familles (cf. fig. 7), en raison à la fois de son nombre toujours élevé de degrés de liberté et de l'homogénéité des variances intra des familles éliminées.

La figure 7 montre également que le carré moyen «famille» et la moyenne générale de la hauteur restent relativement stables lorsque les familles les plus interactives sont progressivement retirées. En ce qui concerne la moyenne, il s'agit d'une conséquence directe de l'indépendance entre interactivité et performance. Pour le carré moyen «famille», ce résultat indique que les familles interactives sont réparties de manière régulière à l'intérieur de la distribution de l'ensemble des familles. Lorsque seules sont conservées les familles les plus stables dans l'espace, les possibilités pratiques de sélection familiale ne sont donc modifiées que par le fait que le nombre de descendances restantes (42 dans le cas particulier) est réduit. 


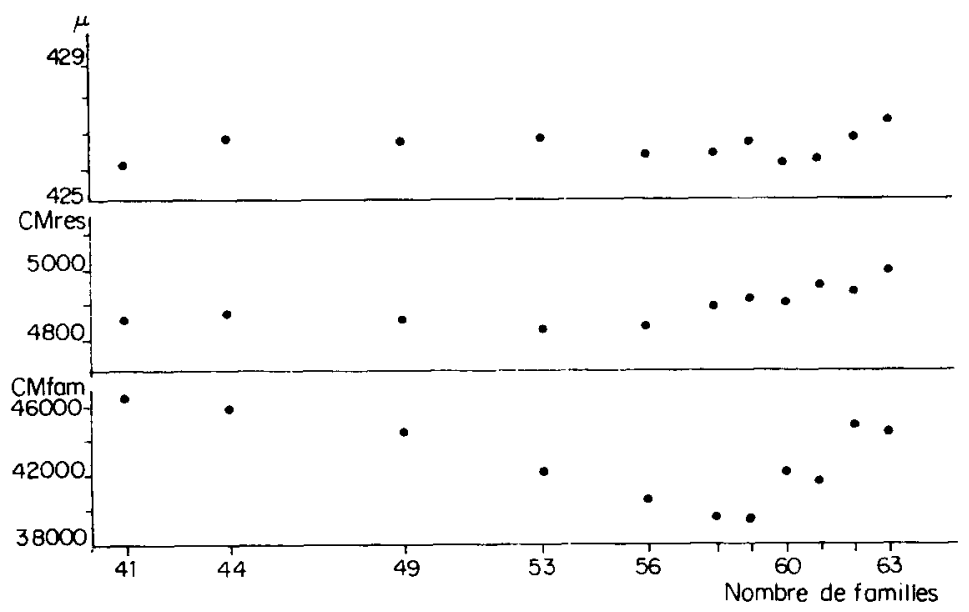

FIG. 7

Evolution de la moyenne générale $(\mu)$, du carré moven " résiduel " (CMres), et du carré moyen "famille " (CMfam) en fonction du nombre de familles classées par interactivité décroissante (de droite à gauche) pour le caractère " hauteur totale 1984 ".

Evolution of grand mean $(\mu)$, error mean square (CMres) and family mean square (CMfam) in terms of number of families. The families are arranged in descending order of total height 1984 relative interactivity (from right to left).

- Application au cas de deux sites.

Une analyse de variance suivant le modèle (3) a été réalisée pour les sites 1 et 2 avec les 63 familles, pour la hauteur. L'évolution du $F$ d'interaction en fonction du pourcentage cumulé d'interactivité a été étudiée grâce au calcul des coordonnées de trois points seulement. Le très bon alignement de ces trois points et la linéarité observée dans le cas des trois sites ont conduit par extrapolation à la droite de la figure 6. Cette dernière appelle trois remarques:

- la valeur du F d'interaction calculé sur 63 familles et pour les sites 1 et 2 est nettement plus faible que pour les trois sites : 1,9 et 3,1 respectivement. Ceci correspond à la forte participation du site 3 à l'interaction globale pour ce caractère (cf. tabl. 5) ;

- la pente de la droite des deux sites est plus faible que celle des trois sites. Sur les sites 1 et 2 , la diminution du $F$ d'interaction en fonction du pourcentage cumulé d'interactivité est donc plus lente ;

- pour rendre la valeur du $\mathrm{F}$ d'interaction sur les deux sites non significative à $5 \mathrm{p}$. 100 , il suffit de retirer sept familles qui totalisent à elles seules $39,4 \mathrm{p}, 100$ de l'interactivité globale.

Connaissant, d'une part, la fonction linéaire entre le $\mathrm{F}$ d'interaction et le pourcentage cumulé d'interactivité, et, d'autre part, l'interactivité relative de chaque famille, il est possible de tracer la courbe correspondant au cas de deux sites, présentée à la figure 5. L'utilisation pratique de cette courbe est identique à celle qui en a été faite dans le cas de trois sites. 


\section{- Interactivité bicaractère}

Dans tout ce qui précède, seul le caractère "hauteur " a été analysé. Des études similaires peuvent être faites pour les trois autres caractères pris indépendamment. Cependant, il peut être nécessaire d'améliorer sur plusieurs caractères simultanément la stabilité par rapport au milieu d'un ensemble de familles.

Les interactivités relatives des différents caractères sont indépendantes les unes des autres. Une interactivité synthétique, définie par la formule (6), a donc été calculée sur les 3 sites pour la hauteur et le débourrement, qui sont les deux principaux caractères pris en considération par les améliorateurs français du Douglas. Par analogie avec la démarche suivie pour un seul caractère, l'évolution des $F$ d'interaction des deux caractères est représentée sur la figure 8 lorsque les familles classées par interactivités relatives synthétiques décroissantes sont progressivement éliminées.

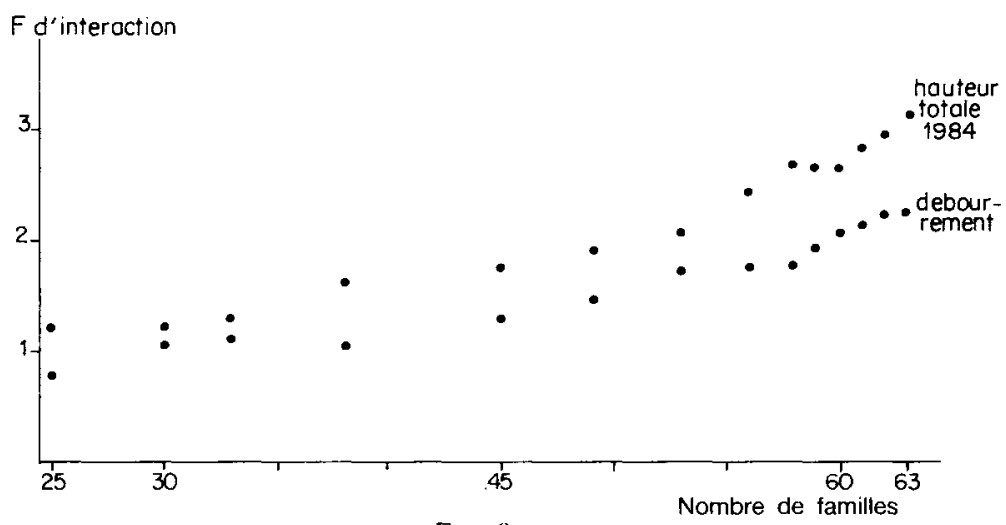

FIG. 8

Evolution du F d'interaction famille $\times$ site pour les caractères " hauteur " et " débourrement ", en fonction du nombre de familles classées suivant l'interactivité synthétique décroissante (de droite à gauche).

Evolution of family $\times$ site interaction Fisher test on "Total height 1984 " and «flushing "traits, in terms of number of families. The families are arranged in descending order of synthetic relative interactivity (from right to left).

La comparaison des figures 5 et 8 conduit à observer que la décroissance du $\mathrm{F}$ d'interaction de la hauteur est à la fois plus irrégulière et plus lente lorsque l'interactivité synthétique est utilisée. De plus, le nombre de familles à éliminer pour rendre la valeur du $F$ d'interaction non significative à 5 p. 100 est de 33 , au lieu de 21 précédemment.

La valeur du F d'interaction pour le débourrement, qui part d'une valeur pour 63 familles moins élevée que celle de la hauteur, atteint plus rapidement le seuil auquel elle n'est plus significative.

Globalement, la forme des deux courbes de la figure 8 est comparable. Cependant, de fortes différences ponctuelles de pente peuvent être observées en raison de l'absence 
de corrélation entre les interactivités des deux caractères (en particulier entre 60 et 58 familles). Inversement l'élimination de quelques familles particulières, dont l'interactivité relative est forte pour les deux caractères, fait parfois évoluer les 2 courbes de manière parallèle (cf. $62^{\mathrm{e}}$ et $61^{\mathrm{c}}$ familles).

Au total, la possibilité de sélectionner un groupe assez nombreux de familles stables pour plusieurs caractères dépend à la fois :

- de l'importance des $\mathrm{F}$ d'interaction pour chaque caractère ;

- de la distribution des interactivités familiales pour chaque caractère ;

- des corrélations entre interactivités familiáles.

\section{Discussion - Conclusion}

Cette étude concerne trois stations expérimentales aux climats et aux conditions édaphiques très variés. L'apparition d'une forte interaction «génotype $\times$ milieu " n'est donc pas surprenante.

Une partie de cette interaction peut être due au niveau provenance, mais le matériel végétal expérimenté ne permet pas d'isoler cette composante. En effet, le peuplement le mieux représenté, La Perrière, ne l'est que par six descendances. Cependant, les provenances artificielles françaises de Douglas incluses dans cette expérience ont démontré par ailleurs une excellente stabilité sur des sites très comparables à ceux étudiés ici (Rosette, 1986). Il semble donc possible d'émettre l'hypothèse que le niveau " descendance maternelle " est principalement responsable de l'interaction "génotype $x$ milieu " mise en évidence.

Cette interaction s'observe en premier lieu sur la vigueur, ce qui était prévisible, et aussi sur d'autres caractères importants pour l'améliorateur et habituellement considérés comme relativement stables dans l'espace parce que soumis à un contrôle génétique plus strict : le débourrement, la flexuosité du fût, l'angle des branches. Dans tous les cas, le carré moyen d'interaction représente de 30 à 45 p. 100 du carré moyen "famille", et il est donc impératif d'en tenir compte dans un schéma d'amélioration.

Deux des voies proposées par FALKenhagen (1985) peuvent être empruntées :

- réunir les sites expérimentaux en groupes à l'intérieur desquels l'interaction famille $\times$ milieu est minimale. Une population d'amélioration et une population de production de graines peuvent alors être sélectionnées et gérées dans chacun de ces groupes. Ainsi, dans l'exemple présenté et pour le: caractère "hauteur ", deux régions d'amélioration pourraient être constituées, l'une pour Peyrat-le-Château et Saint-Girons (sites 1 et 2), l'autre pour Epinal (site 3). Lorsque l'interaction se manifeste fortement entre de nombreux sites, cette démarche a l'inconvénient d'alourdir le travail de sélection par la multiplication des régions d'amélioration ;

- supprimer les familles les plus interactives. Sur les 3 sites analysés, cette solution permettrait d'obtenir un ensemble de 42 familles «stables » vis-à-vis du milieu pour la hauteur. La prise en compte en sus du caractère " débourrement " ferait passer ce nombre à 30 seulement. Si la flexuosité du fût et l'angle de branche étaient également pris en considération, l'effectif final serait encore plus réduit, en raison de l'indépendance des interactivités familiales indiquée au tableau 6. Dans le cas particulier 
de cette étude, les 63 familles de départ formaient donc un ensemble trop limité pour qu'il soit possible de disposer, après suppression des familles instables pour différents caractères, d'un nombre de familles assez grand pour qu'une sélection familiale soit encore efficace. Comme pour toute sélection, l'effectif de la population initiale est donc essentiel pour que l'améliorateur dispose in fine d'un nombre suffisant de génotypes.

Sur le plan méthodologique, le calcul des interactivités apporte de nombreuses informations au sélectionneur, même quand le nombre de sites est faible :

- il permet d'identifier, caractère par caractère, les sites qui participent éventuellement plus fortement que les autres à l'interaction ;

- il permet de classer les génotypes en fonction de leur interactivité pour grouper ceux qui sont stables vis-à-vis du milieu. Cependant la formation de tels ensembles n'est pas toujours possible ni souhaitable, en raison des différents paramètres qu'il faut prendre en compte :

- la distribution des interactivités génotypiques; lorsque toutes les interactivités relatives sont fortement regroupées autour de la valeur moyenne, la sélection de génotypes stables est pratiquement impossible. A l'inverse, celle-ci est d'autant plus facile que se différencie nettement un petit groupe de génotypes ayant de très fortes interactivités relatives;

- l'évolution de la moyenne et de la variance (inter et intra) des génotypes ; ces paramètres peuvent varier dans un sens favorable ou défavorable pour le sélectionneur quand celui-ci élimine les génotypes les plus instables;

- le nombre de génotypes restant après la sélection pour la stabilité, qui doit être suffisant pour que la base génétique soit assez large;

- l'existence éventuelle de génotypes présentant à la fois une forte interaction et des performances bonnes à très bonnes dans certains milieux, dont l'élimination représenterait une perte génétique grave dans ces milieux.

Enfin, lorsque plusieurs caractères sont considérés simultanément, la recherche de génotypes stables vis-à-vis du milieu peut se faire grâce au calcul d'une interactivité synthétique multicaractère. Dans le cas de caractères dont les stabilités sont indépendantes les unes des autres, une simple moyenne pondérée comme celle utilisée ici est suffisante. Dans le cas contraire, les interactivités élémentaires peuvent être transformées par une analyse en composantes principales en combinaisons linéaires d'interactivités corrélées, comme le suggèrent BaRADAT \& YAZDANI (1987). En général, le nombre de génotypes stables finalement retenus par une telle approche sera plus faible que dans le cas d'un seul caractère.

Reçu le 3 juin 1986.

Accepté le 25 novembre 1986. 


\section{Summary \\ Family $\times$ site interaction in a three-site progeny-test with Douglas-Fir (Pseudotsuga menziesii (Mirb.) Franco)}

\section{Material and Meihods}

The results given in this paper come from a multi-site provenance-progeny test with artificial french origins of Douglas-Fir ( 63 open-pollinated progenies). The ecological features of the three field trials are very different (fig. 1), each being however considered «normal " for this species. Six traits are taken into account, most of them assessed at age 7 in the field, i.e. 10 years after the seed : total height, flushing, stem sinuosity, branch angle, forking, lamma shoot frequency (fig. 2 ). The aim is to reveal family $\times$ site interaction and to find a practical way of selecting stable-inspace progenies.

Analysis of variance is performed at within and between-sites levels (models 1,2,3). Between sites, the contribution of each site and family to the overall interaction sum of squares is evaluated through its relative interactivity defined as the inverse of WRICKE's ecovalence (1962) (models 4 and 5). A synthetic interactivity is proposed to evaluate family stability for some traits simultaneously (model 6) when single-trait interactivities are independant.

\section{Results}

- Site by site (tabl. 2 and 3). From one site to another, large differences appear on grand means, family-effect $\mathrm{F}$-values and between-tıaits correla:ions.

- Overall, highly significant family $\times$ site interactions are found on total height, flushing, stem sinuosity and branch angle (tabl. 4). On the two other traits, interaction is not significant.

- Relative interactivity of site 1 is generally the lowest, whereas that of site 3 is among the highest (tabl. 5).

- For cach trait, a limited group of progenies the interactivities of which are high is revealed on figure 3. Relative interactivities of families are independant from each other (tabl. 6) and from family-means for the same trait as well (fig. 4). On figure 5 , it is shown how family $\times$ site interaction on total height is reduced when the most interactive families are removed one by one. When 42 families only are left, interaction F-value is no longer significant at 5 p. 100 level. When the number of families is reduced, total height grand mean, error and family mean squares are not much affected as shown on figure 7.

- Families are arranged and removed in descending, order of a synthetic interactivity on total height and flushing, which are the main traits interesting the French Douglas-Fir breeders. The corresponding evolution of interaction F-values on each trait is shown on figure 8 . To bring both F-tests to non significant values at $5 \mathrm{p}$. 100 level, it is necessary to remove 33 progenies, so that only 30 are left. Such a limited group of families is considered too small for further selection.

\section{Discussion - Conclusion}

On this sample of Douglas-Fir progenies, a large family $\times$ site interaction is detected. This interaction affects total height, which is no surprise, and also flushing, stem sinuosity and branch angle which were up-to-now considered less sensitive to site effect.

Both choices offered by FalKenHagen (1985) to deal with G-E interaction are discussed. Finally, methodological usefullness in tree breeding of interactivities derived from WRICKE'S ecovalences is emphasized, and some parameters worth looking at are listed.

Key words: Genotype by environment interaction, half-sibs progeny, height growth, flushing, stem form, branch angle, Pseudotsuga menziesii. 


\section{Références bibliographiques}

Bachacou J., Masson J.P., Millier C, 1981. Manuel de la programmathèque statistique AMANCE 81, Département de Biométrie. Institut National de la Recherche Agronomique, Copedith.

Baradat Ph., 1982. Génétique quantitative. Modèles statistiques el génétiques de base, Fascicule 1. I.N.R.A. Laboratoire d'Amélioration des Arbres Forestiers - Bordeaux. Document interne.

BARAdat Ph., Yazdanı R., 1987. Stability of genotype expression for monoterpenes synthesis in clones of Scots Pine grown in different stations. (Accepté pour publication). Scand. J. For. Res.

Denis J.B., Vincourt P., 1982. Panorama des méthodes statistiques d'analyse des interactions génotype $\times$ milieu. Agronomie, 2 (3), 219-230.

FALCONER D.S., 1974. Introduction à la génétique quantitative. Masson et $\mathrm{C}^{\mathrm{ie}}$.

Falkenhagen E.R., 1985. Genotype by environment interactions in South African pine progeny trials : implications for tree breeding. South African Forestry Journal, 135, 53-60.

Freeman G.H., 1973. Statistical methods for the analysis of genotype $\times$ environment interactions. Heredity, 31 (3), 339-354.

RosetTE C., 1986. Contribution à l'exploration de la variabilité infraspécifique du Douglas (Pseudotsuga menziesii, Mirb. Franco). Mémoire de $3^{e}$ année ENITEF. I.N.R.A. Station d'Amélioration des Arbres Forestiers. Document interne $n^{\circ} 345 / 86$.

SKRфPPA T., 1984. A critical evaluation of methods available to estimate the genotype $\times$ environment interaction. Studia Forestalia Suecica, 166, 3-14.

WRICKE G., 1962. Uber eine Methode zur Erfassung der okologischen Streubreite in Feldversuchun. Z. Plfanzenzchutz, 47, 92-96. 